\title{
INTEGRAL ADAPTIVE AUTOPILOT FOR AN UNMANNED AERIAL VEHICLE
}

\author{
Volodymyr GRITSENKO, Oleksandr VOLKOV, Mykola KOMAR, Dmytro VOLOSHENYUK \\ International Research and Training Center for Information Technologies and Systems of National Academy of \\ Sciences of Ukraine and Ministry of Education and Science of Ukraine, Kyiv, Ukraine
}

Received 27 July 2018; accepted 23 October 2018

\begin{abstract}
The aim of research is to study the modern algorithms used in autopilots of unmanned aerial vehicles and formulation of the problem of development and usage of new intellectual methods for automatic control systems. The approach considered in the article is based on the theory of high-precision remote control of dynamic objects and on the complex interaction of methods of theory of invariance, adaptive control and intellectualization of processes of UAV control. One of the features of the proposed method of intellectual control for unmanned aerial vehicle autopilot is the procedure of transforming a multi-dimensional system into an aggregate of virtual autonomous processes, for each of which the control algorithm is easily generated by an autonomous subsystem. Coming up next is the procedure of coordination of actions of all the autonomous systems into single functioning complex. This provides an opportunity to improved precision and sustainability of control. Using the method described in the article allows creating integral and adaptive autopilots to perform complicated spatial maneuvering an unmanned aerial vehicle being based on usage of full non-linear models without simplifications and linearization.
\end{abstract}

Keywords: unmanned aerial vehicle, control system, invariance, virtual control, autopilot, method, adaptation.

\section{Introduction}

At present unmanned aerial vehicles (UAVs) are successfully used in various industries in performing scientific and engineering, economical, military and a number of other missions. Effectiveness of their functioning is mainly determined by an onboard suit of hardware and software of a UAV's control system, which, as a rule, is done by means of integrated avionics being based on a single computing platform. The automatic control system (ACS) mission is to provide the required dynamic precision within a wide range of aircraft parameters change.

The modern way of onboard control systems development reckons for control algorithms implementation using programmed modules written in high level programming languages based on the microprocessors of computing capacity being enough for the algorithms, in the form of a UAV's single autopilot hardware and software suit, to be realized (Fahlstrom \& Gleason, 2012).

The existing UAV automatic control systems perform UAV flying tasks, among which the following may be highlighted:

- fulfilling the controlled parameter commands;
- controlling intended to stabilize the preset parameters of an aircraft and its energy data;

- performing UAV autonomous flight through the specified way points and into the targeted air spaces in automatic mode without pilot-operator's interference.

AP detects deviation of the actual UAV flight trajectory from its programmed one and, according to the deviation value, generates commands to correct the UAV actual trajectory and controls the position and the direction of the UAV in the space throughout all the flight stages as well.

The process of the existing autopilot systems enhancement is intended to broaden the range of UAV's tasks without direct human involvement and introduce additional smart functions into autopilot operation.

\section{Methodology and theoretical background}

In designing UAV ACSs, automatic control theory methods are used together with usage of calculus of variation, optimal control, mathematic programming, game theory, intellectual control, flight data processing methods and etc. (Moiseyev, 2013).

*Corresponding author. E-mail: nickkomar08@gmail.com 
A UAV is a dynamic object with a big number of parameters and complex cross interfaces between them and described with the essentially nonlinear differential equations, simplification or linearization of which in solving complicated tasks with steep maneuvering of a UAV is not admissible. In addition, the common feature of the UAV control tacks, regardless of their types and applications, is the need to consider various evidences of uncertainty, the main sources of which are the following factors (Beardand \& McLain, 2012):

- indistinctness of functioning objectives and control tasks;

- non-stationarity of UAV parameters and its control systems;

- uncertainty of situation and flight plan performance conditions;

- presence of random ambient environment influences;

- incoming information deterioration in remote data transfer channels.

The following conceptions have been laid down in the theoretical basis of the UAV intellectual control method, proposed the article authors:

1. nonlinear controlled objects full description conception and dynamically changing ambient environment, that allows to evaluate and use not only the present technological resource but the hidden full system potential;

2. control conception, that is realized by availability of the applied knowledge base with structural adaptation, that makes high precision UAV control:

- with various uncertainty levels and limitations;

- with integration of various navigation data sources;

- with computational complexity that is optimal for the tasks assigned and specified communicational limitations;

3. tactical situations development recognition conception as a base for decision making in the conditions that are featured with high degree of uncertainty in the current navigation situation.

Besides, the scientific platform of the proposed intellectual control system is based on the theory of high precision remote control of dynamic objects in the condition of uncertainty and complicated limitations. Effectiveness of the theory is achieved with complex interaction of the invariant theory, autonomy, adaptive virtual control as well as new engineering solutions, created by the staff of the article authors. Joint usage of theoretical and conceptual bases allows generating fundamentally new control algorithms for a UAV's autopilot that provide:

- high quality and stability of the controlled object while making complicated spatial flight trajectories with significant changes of flight altitude and speed and presence of essentially curved maneuvering segments in horizontal and vertical planes;

- invariance to outer disturbances and adaptability to parametric uncertainties.

\section{Review of the control methods of modern UAV's autopilots}

The present article is dedicated to the modern algorithms study aspects that are used in UAV's AP and formulation of the problem of generation and usage of intellectual methods and UAV automatic control systems as well as description of the UAV intellectual control method proposed by the authors.

Development of information technologies allows using complicated intellectual control methods even in relatively cheap in cost APs. Nowadays, there is a big number of ACSs for UAVs of different types and assignments, which differ from one another by both costs and algorithm structures used in them (Chao, Cao, \& Chen, 2010). But in a number of cases, there are the APs spread out that are positioned as universal control means for a wide range of UAVs and remain insufficiently effective since they don't fully take into account the structural features of a given UAV as well as essential UAV parameters nonlinearity in steep maneuvering.

Therefore, the forthcoming development of algorithms of UAV ACS is held with the purpose of covering still bigger range of decision making situations in the condition of significant uncertainty and approximating (and under certain parameters exceeding) UAV automatic control system performance to skilled pilot-operator behavior.

The modern UAV ACS designs are based on usage of both conventional proportional-integral differential (PID) controllers and prospective approaches: adaptive neuron networks, optimizing methods, fuzzy logic etc. (Feng, 2006). Below are some of them considered:

\section{Approach to UAV control based on PID controllers}

In the source (Shilov, 2014), the proportional-integral cascade (PI-cascade) (shown in the figure1 below) being applied to each axis of rotation (bank, pitch and yaw) is used as an automatic stabilization algorithm for UAV's angles of orientation as well as for flight altitude control.

In the Figure 1: $\alpha_{a c t}$ - UAV's current angle of orientation, $\alpha_{\text {des }}$ - UAV's desired angle of orientation, $\alpha_{\text {err }}-$ angle of orientation error, $\Omega_{\text {des }}$ - desired angular velocity of rotation about an axis, $\Omega_{\text {act }}$ - current angular velocity of rotation about an axis, $\Omega_{\text {err }}$ - error of angular velocity of rotation about an axis, out Out - outcoming signal to UAV's control actuators. The results of computer modeling of performance (Shilov, 2014) have shown, that an AP based on conventional PID controllers does not exclude

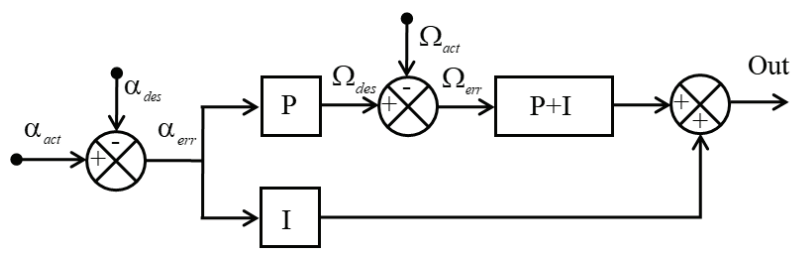

Figure 1. PI-cascade diagram for stabilization control of angle of orientation 
existence of a control error caused by perturbing influence and does not allow to provide required dynamic precision of control of the angle of orientation while changing flight modes and ambient conditions.

In their turn, the ACSs built on PID controllers have a number of advantages such as:

- simplicity and easiness of the controllers designing;

- low requirements to computing resources;

- simple control algorithms structure and relatively simple calculation of the controller coefficients.

Along with the advantages the ACSs have the following flaws:

- PID controller's parameters resetting is needed when controlled object data are changed;

- widespread methods of controller's settings, such as, for instance, intuitive setting, characteristic method, analytic method can't provide high quality control due to inability to change the controller's data while in the process of influence by disturbance and absence of PID controller parameters dynamic change;

- calculating and setting the ACS controllers coefficients require significant correction at early stages of tests and operation, and, in a number of cases, admit unsatisfactory flight performance data.

So, incorporation of PID controllers in non-linear systems without their being added with more sophisticated control strategies that compensate their disadvantages, put restrictions on reliability and precision of such control systems.

\section{Approach to UAV control based on neural networks}

UAV ACSs based on the adaptive neural network controller doesn't require a precise mathematical model, which is evident advantage of such ACS and suitable for multipurpose UAVs control (Calise \& Rysdyk, 1998). An example of such ACS for controlling UAVs is proposed in (Johnson \& Kannan, 2002). The synthesis of the controller is made with division into the outer loop and the inner loop (shown in the Figure 2). The outer loop controls UAV orientation by changing moments that act upon the UAV with control surfaces position changing. The outer loop controls the forced that act upon the UAV by changing thrust value and the UAV's orientation with engaging the inner loop.

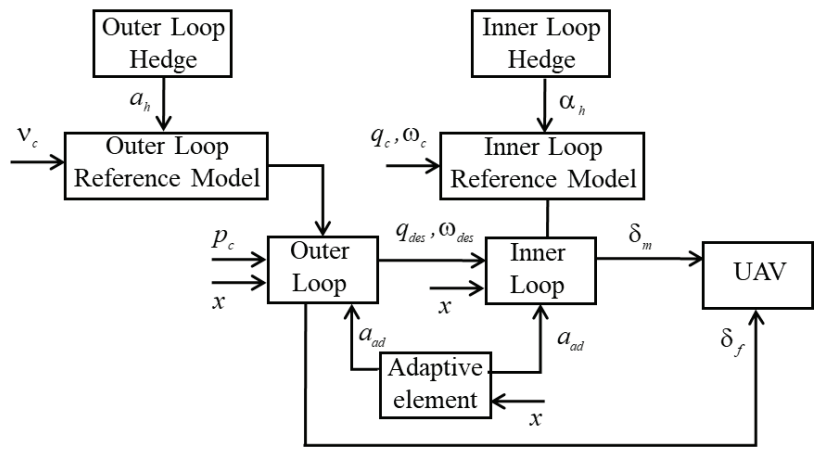

Figure 2. Inner and outer loops of ACS with adaptation
In the Figure2: $x$ - position vector, $p$ - UAV position, $v$ - pseudo control vector, $a$ - acceleration, $\alpha$ - angular acceleration, $q-$ quaternion, $\omega-$ angular velocity, $\delta-$ control vector (control surfaces deflection), lower indexes, $c$-command signal, $h$ - limitations, $a d$ - adaptive signal, des - desired signal, $m$ - moment, $f$ - force.

Parametric uncertainty, which appears in the course of controlling a UAV, is minimized by using the adaptive element. Neural network (perceptron with one hidden layer as a universal approximator) is used as an adaptive element by Johnson and Kannan (2002).

Among the disadvantages being proper to the systems of such type, one should mark algorithm structure complexity, unwanted adaptation to the input parameters of the controlled object such as dynamics of actuators, as well as long duration of the neural network study process to achieve high control precision, which, in its turn, decreases with emerging casual disturbance.

\section{Approach to UAV control based on optimization methods}

In the works of Lopez, R. Dormido, S. Dormido, and Gomez (2015) and Lopez, R. Dormido, Gomez, S. Dormido, and Diaz (2007), there is proposed control architecture for the inner and outer loops of ACS for UAV movement, which is realized with using an $\mathrm{H} \infty$-controller. An ACS consists of two loops: inner controller loop to provide robustness for parametric uncertainty and outer loop for tracking the reference model (shown in the Figure 3).

The inner loop structure is intended to control the angles of pitch, bank, yaw as well as the engine thrust.

Two different controllers refer to the outer loop: controllers of altitude and course. Both controllers are synthesized by using $\mathrm{H} \infty$ optimization.

The modeling results that are presented by Lopez et al. (2015) show that using $\mathrm{H}_{\infty}$ controller doesn't provide the control with required parameters of stability and quality with simplifying the controlled object model. So, they set high requirements for quality, completeness and precision of the reference mathematical models. As a rule, optimization requires a significant number of computing resources and much time. Using $\mathrm{H} \infty$ controller to control a non-linear object involving simplified linearized controlled object model causes parametric uncertainty.

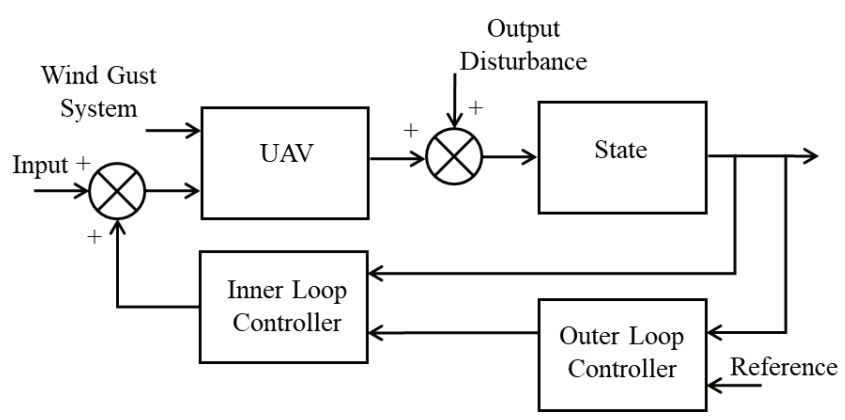

Figure 3. UAV ACS architecture based on optimization method 


\section{Approach to UAV control based on fuzzy logic}

Fuzzy logic control systems can be used in many fields including UAV flight control. Together with studies of other modern control strategies, a plenty of works are dedicated to incorporation of fuzzy logic controllers into APs (Ross, 2004). Conventional fuzzy logic control system structure consists of four basic components: knowledge base, interface for converting discrete set into fuzzy one, derivation mechanism and interface for converting fuzzy set into discrete one. The block diagram for a fuzzy logic control system is shown in the Figure 4 below.

Synthesis of UAV's autopilot with usage of fuzzy logic is given in work of Kumon et al. (2006). The AP consists of three controllers based on fuzzy logic (one controller is used for controlling UAV's side movements, two of them for controlling lateral movement). To improve precision of control the velocity control unit and the cancelation unit for disturbances caused by wind gusts have been added to the autopilot. The proposed system structure is given in the Figure 5.

According to the results of modeling, given by $\mathrm{Ku}$ mon et al. (2006), it is understood that in presence of disturbances a UAV not always comes up to the specified waypoint which results in trajectory curvature and extra maneuvers and imprecisions in holding specified flight altitude emerge as well.

The common disadvantages of the control system based on fuzzy logic methods include design complexity of the systems of this kind, extended process of tuning and

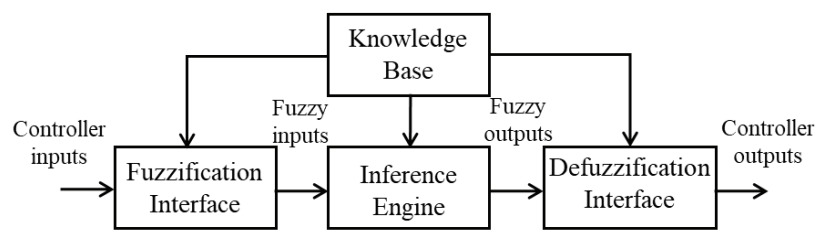

Figure 4. Basic control system structure with fuzzy logic

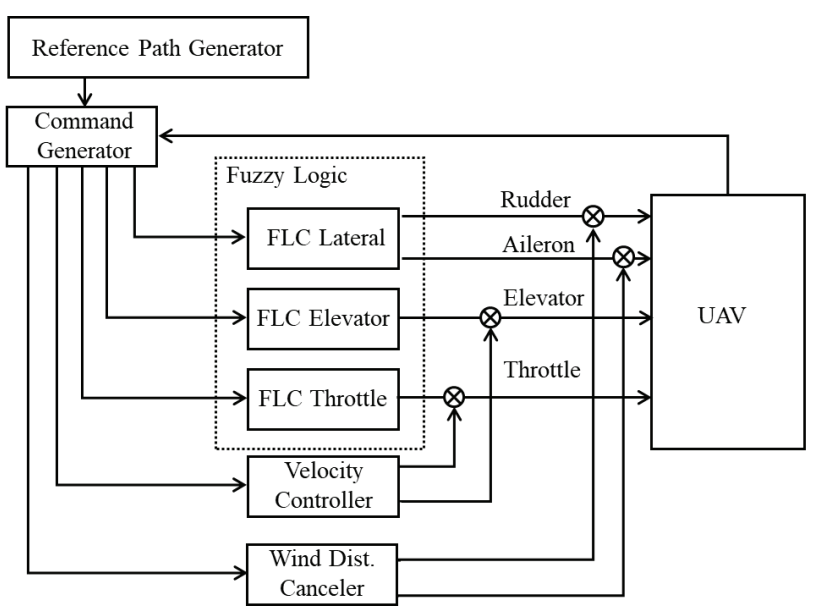

Figure 5. Block diagram for fuzzy logic autopilot control system analysis of adequacy of the designed real model system, high requirements for the rule bases for the fuzzy system realized.

From what has been said above emerges that the control systems of the majority of unmanned aerial vehicles being built on the basis of the approaches mentioned above have limited performance capabilities focused on automatic or semiautomatic operation modes in specified conditions. Utilizing conventional intellectual control methods without extra actions as for their performance quality improvement doesn't provide required precision and autonomous control. Therefore, a new approach to the issues of effective control of UAVs of different purposes in various and unexpected conditions is needed.

\section{Development and description of integral adaptive autopilot}

To eliminate the flaws of the abovementioned methods of building UAV's ACSs, the authors propose the new method and algorithm of integral and adaptive autopilot. High performance of the modern integrated avionics suits enables, in real time, to carry out, directly on board the UAV, both coefficient adjustments and reconfiguration of the control system structure, thereby improving quality and stability of the control process for a wide range of flight assignments. Reckoning for that, the authors have proposed the integral and adaptive autopilot (shown in the Figure 6) that enables removing a number of flaws of the modern UAV's ACSs, in particular:

- to compensate cross connections and mutual influence between the control channels;

- to provide for generation of integral and adaptive control commands through all the channels and to achieve improved quality and stability of controlling while performing complex spatial trajectories with big changes of the flight altitude and velocity, as well as in presence of essentially curved segments of maneuvering in vertical and horizontal planes;

- to achieve the UAV control precision improvement in presence of outer and inner disturbances.

The structural diagram of the integral and adaptive autopilot contains: generator of given control parameters, roll error formation unit, pitch error formation unit, sensor unit, coordinated turn calculating unit, roll virtual control dynamics unit, yaw virtual control dynamics unit, pitch virtual control dynamics unit, aerodynamic data storage and approximation unit, linear computing unit and nonlinear computing unit.

Such structure has been chosen to realize UAV control in the class of virtual strategies (Pavlov \& Pavlova, 2015). In this case, virtual control acts as a conventional control virtualization method with usage of PID controllers (Grytsenko, Volkov, Komar, \& Bogachuk, 2018).

The virtual control (VC) allows studying the controlled object comprehensively without simplifications and linearization of equation systems describing it (Pavlov \& Pavlova, 2015). 


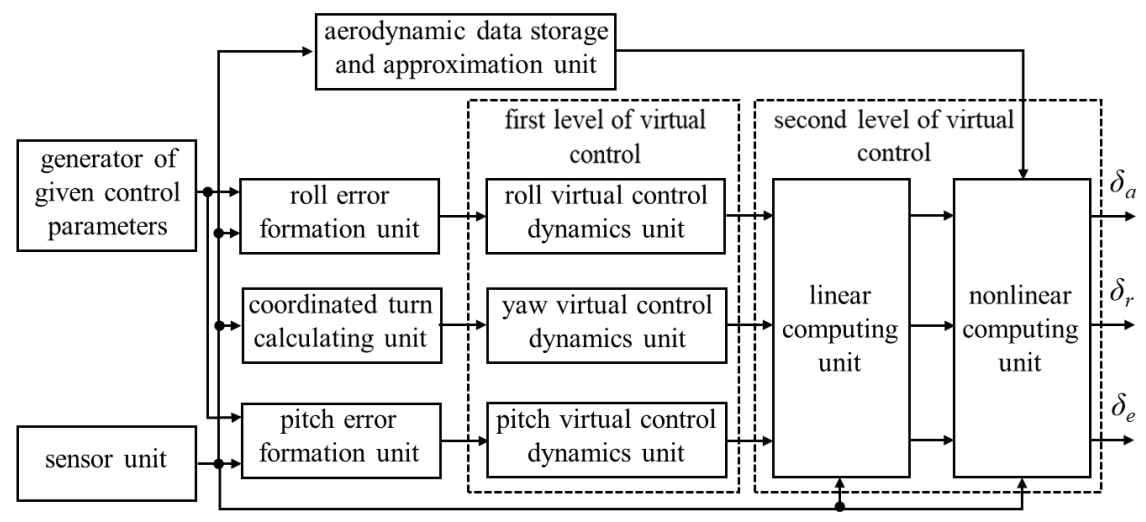

Figure 6. Structural diagram of integral and adaptive UAV's autopilot

In this case, $\mathrm{VC}$ envisages the problem solving in two stages.

The first stage - to synthesize a virtual object with invariant and autonomously organized control being based on the original real object.

The second stage - to synthesize the real control of a real object after the virtual object and the virtual control.

The first stage's objective is synthesis of a virtual object and searching for the area of possible control of the virtual object, which enables to solve the problem of synthesis of the real object control in the second stage being based on the virtual control.

In the structural diagram, the units forming the layers of virtual control are marked with a dotted line. The real commands for aileron deflection angles $\delta_{a}$, elevator $\delta_{e}$ and rudder $\delta_{r}$ are generated at the output of the nonlinear computing unit.

Differential equation system that describes the virtual object has, in comparison with the output equation system that describes the real object, redundant solutions, and the output equation system's solutions are simultaneously the solutions of the new system. Insuring invariant and autonomous conditions for all the array of the new system's solutions will automatically insure invariance and autonomy for the output equation system's solutions.

Usage of $\mathrm{VC}$ allows considering full non-linear models of objects without admitting simplifications and linearization. Using such, an approach allows to design fundamentally new non-linear controllers that ensure stability of the controlled objects as well as invariance to outer and inner disturbances.

Accordingly, the designed UAV integral and adaptive spatial maneuvering control autopilot works in the following way to insure.

The generator of given control parameters cyclically generates the time sequence of the command signals for roll $\gamma_{c m d}$ and pitch $\vartheta_{c m d}$ to insure targeted spatial maneuvering in horizontal and vertical planes respectively.

At the sensor unit output the UAV's current state signals, such as the sensor signals for roll $\gamma$, yaw $\psi$, pitch $\vartheta$, the sensor signal for air temperature $T$, the sensor signal for barometric pressure $B$, the sensor signal for air speed, the sensor signals for accelerations $n_{x}, n_{y} n_{z}$ and angular velocity signals $\omega_{x}, \omega_{y} \omega_{z}$ are generated in the UAV associated coordinate system $O X Y Z$, origin of which is places in the UAV's center of mass, the $O X$ axis lies in the UAV's plane of symmetry and directed to the UAV's nose part, the $O Y$ axis lies in the UAV's plane of symmetry and directed upwards (in horizontal flight), and the $0 Z$ axis adds the system to the right.

The error formation units for roll and pitch, after the command roll $\gamma_{c m d}$ and pitch $\vartheta_{c m d}$ signals that come from the generator of given control parameters, generate the signals of the form $\Delta \gamma=\gamma_{c m d}-\gamma$ and $\Delta \vartheta=\vartheta_{c m d}-\vartheta$ respectively. The signals for the current roll angle $\gamma$ and pitch $\vartheta$ come from the sensor unit. Then the error signals for roll and pitch come to the virtual control dynamic units for roll angle and pitch.

The signals of roll angle $\gamma$, air speed sensor $V$, side acceleration $n_{z}$, and angular velocity $\omega_{y}$ about the $O Y$ axis from the sensor unit are sent to the coordinated turn calculated unit, which, in order to ensure a UAV coordinated turn without sideslip, generates the error signal for the coordinated turn angular $\Delta \omega_{y}$ velocity and the error signal for the side acceleration in the following way:

$$
\begin{aligned}
& \Delta \omega_{y}=K_{R Y} \frac{g}{V} \sin \gamma-\omega_{y} ; \\
& \Delta n_{z}=K_{n} n_{z},
\end{aligned}
$$

where $g$ - acceleration of gravity, $K_{R Y}$ - coordinated turn displacement, $n_{z}$ - side acceleration current value. The error signals for the coordinated turn angular velocity $\Delta \omega_{y}$ and the error signals for the side acceleration $\Delta n_{z}$ from the coordinated turn calculation unit come from the yaw virtual control dynamics unit.

The roll virtual control dynamics unit, the yaw virtual control dynamics unit and the pitch virtual control dynamics unit collectively constitute the first level of virtual control for UAV's spatial maneuvering. These units give the idea of multidimensional control system in the form of a complex of independent one-dimensional autonomous control systems for roll $p_{\gamma}$, yaw $p_{\psi}$ and pitch $p_{9}$ : 


$$
\begin{aligned}
& \ddot{\gamma}=p_{\gamma} ; \\
& \ddot{\psi}=p_{\psi} ; \\
& \ddot{\vartheta}=p_{\vartheta},
\end{aligned}
$$

where $p_{\gamma}, p_{\psi}, p_{\vartheta}-$ command signals of virtual control of roll, yaw and pitch the unit's outputs. With this presentation of the system (1) for these autonomous relations, they can use all the design methods that are developed for one-dimensional systems, such as, for instance, the methods for building proportionate integral differential controllers, the methods for building speed-optimized controllers and others. By adding the control algorithms using PID controllers with virtual control, one can achieve higher quality of control by eliminating the PID controller's deficiencies.

The roll virtual control dynamics unit, the yaw virtual control dynamics unit and the roll virtual control dynamics unit compute the command signals for virtual control of roll $p_{\gamma}$, yaw $p_{\psi}$ and pitch $p_{\vartheta}$ by the following relations:

$$
\begin{aligned}
& p_{\gamma}=\left(K_{\gamma}+\frac{1}{p T i_{\gamma}}+p T d_{\gamma}\right) \Delta \gamma \\
& p_{\vartheta}=\left(K_{\vartheta}+\frac{1}{p T i_{\vartheta}}+p T d_{\vartheta}\right) \Delta \vartheta ; \\
& p_{\psi}=\left(K_{\omega_{y}}+\frac{1}{p T i_{\omega_{y}}} p T i_{\omega_{y}}+p T d_{\omega_{y}}\right) \Delta \omega_{y}+ \\
& \left(K_{n_{z}}+\frac{1}{p T i_{n_{z}}} p T i_{n_{z}}+p T d n_{z}\right) \Delta n_{z},
\end{aligned}
$$

where $p=\frac{d}{d t}$ - time differentiation operator, $K_{\gamma}, T d_{\gamma}$, $T i_{\gamma}$ - the coefficients for the proportional, integral, and derivative terms of the PID controller in the roll channel, $K_{\vartheta}, T d_{\vartheta}, T i_{\vartheta}$ - the coefficients for the proportional, integral, and derivative terms of the PID controller in the pitch channel, $K_{\omega_{y}}, T d_{\omega_{y}}, T i_{\omega_{y}}$ - the coefficients for the proportional, integral, and derivative terms of the PID controller in the yaw channel for the angular velocity error of the coordinated turn $\Delta \omega_{y}, K_{n_{z}}, T d n_{z}, T i_{n_{z}}$ - the coefficients for the proportional, integral, and derivative terms of the PID controller in the yaw channel for the side acceleration $\Delta n_{z}$.

Kinematic relations (taking into account admission of ignorance of influence of the local vertical orientation change and horizontal plane within the time the UAV's movement relatively to the spherical Earth), which combine the derivatives of the angles $\dot{\psi}, \dot{\vartheta}, \dot{\gamma}$ with the angular velocities of rotation of the UAV, have the following form:

$$
\begin{aligned}
& \dot{\psi}=\frac{\left(\omega_{y} \cos \gamma-\omega_{z} \sin \gamma\right)}{\cos \vartheta} ; \\
& \dot{\vartheta}=\omega_{y} \sin \gamma+\omega_{z} \cos \gamma ; \\
& \dot{\gamma}=\omega_{x}-\left(\omega_{y} \cos \gamma-\omega_{z} \sin \gamma\right) \frac{\sin \vartheta}{\cos \vartheta} .
\end{aligned}
$$

The system of relations of the first level virtual control for spatial maneuvering (1) and the kinematic equations system (2) allow to form the system of three linear equations with three unknown command signals of the second level virtual control $\dot{\omega}_{x C}, \dot{\omega}_{y C}, \dot{\omega}_{z C}$ :

$$
\begin{aligned}
& k_{1 x} \dot{\omega}_{x C}+k_{1 y} \dot{\omega}_{y C}+k_{1 z} \dot{\omega}_{z C}=K_{1} ; \\
& k_{2 x} \dot{\omega}_{x C}+k_{2 y} \dot{\omega}_{y C}+k_{2 z} \dot{\omega}_{z C}=K_{2} ; \\
& k_{3 x} \dot{\omega}_{x C}+k_{3 y} \dot{\omega}_{y C}+k_{3 z} \dot{\omega}_{z C}=K_{3} .
\end{aligned}
$$

Coefficients $k_{1} x, k_{1} y, k_{1} z, k_{2} x, k_{2} y, k_{2} z, k_{3} x, k_{3} y, k_{3} z, K_{1}$, $K_{2}, K_{3}$ in the equation system (3) that are computed by differentiating the relations in (2), have the following values:

$$
\begin{aligned}
& k_{1 x}=0, k_{1 y}=\frac{\cos \gamma}{\cos \vartheta}, k_{1 z}=\frac{-\sin \gamma}{\cos \vartheta} ; \\
& k_{3 x}=1, k_{3 z}=\frac{\sin \gamma \sin \vartheta}{\cos \vartheta} ; \\
& k_{2 x}=0, k_{2 y}=\sin \gamma, k_{2 z}=\cos \gamma ; \\
& K_{1}=p_{\psi}+\omega_{y}\left[\left(\frac{\sin \gamma}{\cos \vartheta}\right)-\left(\frac{\cos \gamma \sin \vartheta}{\cos ^{2} \vartheta}\right)\right]+ \\
& \omega_{z}\left[\left(\frac{\cos \gamma}{\cos \vartheta}\right)-\left(\frac{\sin \gamma \sin \vartheta}{\cos \vartheta}\right)\right] ; \\
& K_{2}=p_{\vartheta}-\omega_{y} \cos \gamma+\omega_{z} \sin \gamma ; \\
& K_{3}=p_{\gamma}-\omega_{y}\left[\left(\frac{\cos \gamma}{\cos ^{2} \vartheta}\right)-\left(\frac{\sin \gamma \sin \vartheta}{\cos \vartheta}\right)\right]- \\
& \omega_{z}\left[\left(\frac{\cos \gamma \sin \vartheta}{\cos \vartheta}\right)+\left(\frac{\sin \gamma}{\cos ^{2} \vartheta}\right)\right] .
\end{aligned}
$$

According to the virtual control signals for roll $p_{\gamma}$, yaw $p_{\psi}$ and pitch $p_{\vartheta}$, the linear computing unit, by solving the equations (3) allowing for the relations (4) and the UAV's current state signals, generates the second level virtual control command signals:

$$
\begin{aligned}
& w_{x}=\dot{\omega}_{x C} ; \\
& w_{y}=\dot{\omega}_{y C} ; \\
& w_{z}=\dot{\omega}_{z C},
\end{aligned}
$$

which are intended to form afterwards the real control commands for the deflection angles ailerons, elevator and rudder directly.

The second level virtual control signals from the linear computing unit come to the non-linear computing unit, to the input of which the UAV's current state signals come from the output of the sensor unit.

The non-linear computing unit solves the equation system the UAV's movement relatively to the center of mass in the associated coordinate system. Possible variants of writing down the non-linear differential equation systems are given by Kharchenko, Chepizhenko, Tunik, and Pavlova (2012) and Bodner (1973). To solve the equation systems, the non-linear computing unit can use any of the known non-linear equation system computing methods, for instance: the methods of Newton-Raphson, Seidel, Broyden and others. The more precisely the used relations describe the real parameters of UAV movement, the more 
effective the process of autopilot integral adaptation to the maneuvering real conditions will be.

The coefficients for aerodynamic forces and moments needed for calculations are in the aerodynamic data storage and approximation unit in the form of data tables with coefficients of aerodynamic forces and moments that are generated based on the aerodynamic study the relevant UAV.

Based on the second level virtual control signals $w_{x}$, $w_{y}, w_{z}$ the non-linear computing unit, on the basis of the linear equations solution and allowing for the signals of aerodynamic forces and moments coefficients that are coming from the aerodynamic data storage and approximation unit and the UAV's current state signals that are coming from the censor unit, generates the real control commands for the deflection angles of ailerons $\delta_{a}$, elevator $\delta_{e}$ and rudder $\delta_{r}$, which are afterwards sent to the UAV's control surfaces actuators in the form of angles of their deflection.

The developed autopilot has the following advantages:

- it processes the cross interfaces and compensate mutual influence between the UAV control channels;

- it provides for integral adaptation of the control commands that were generated for all the control channels;

- it improves precision and increase reliability of performing complicated spatial flight trajectories;

- it allows to adapt to essential changes in flight altitude and speed of the unmanned aerial vehicle;

- it enables maneuvering in vertical and horizontal planes with essentially curved segments;

- it increases quantity and enhance effectiveness of the alternate ways of a UAV movement planning.

\section{Conclusions}

Most of the works that dedicated to the intellectual adaptive control are based on the linear or essentially simplified non-linear mathematical models of a controlled object. In its turn, the problem of intellectual control of a class of non-linear complex systems remains an opened and crucial issue.

Solving the issues for effective controlling UAVs of different purposes in various and unpredictable conditions changing fast, requires new approaches to synthesis of non-linear controllers. One of the modern tools for solving the issues is incorporating intellectual control systems into the prospective UAVs.

The studies carried out have proven insufficient effectiveness of the conventional UAV control systems performance in presence of outer disturbances and in case of steep UAV maneuvering.

Using the method described in the article allows creating integral and adaptive autopilots to perform complicated spatial maneuvering an unmanned aerial vehicle being based on usage of full non-linear models without simplifications and linearization. This provides an opportunity to use broadened range of parameters and alternative options for on-board systems structural organization as well as improved precision and sustainability of control. One of the points of the method is the procedure of transforming a multi-dimensional system into an aggregate of virtual autonomous processes, for each of which the algorithm is easily generated an autonomous subsystem. Coming up next is the procedure of coordination of actions of all the autonomous systems into single functioning complex.

The developed laws of adaptive control of UAV movements are synthesized on the basis of invariance and autonomy. These laws meet the hybrid system requirements and enable controlling UAVs in the conditions where their movements are described by non-linear dynamic equation systems.

\section{References}

Beardand, R. W., \& McLain, T. W. (2012). Small unmanned aircraft: theory and practice. Princeton: Princeton University Press.

Bodner, V. (1973). Sistemy upravleniya letatel' nymi apparatami. Moscow: Mashinostroyeniye.

Calise, A., \& Rysdyk, R. (1998). Nonlinear adaptive flight control using neural networks. Control Systems Magazine, 18(6), 14-25.

Chao, H., Cao, Y., \& Chen, Y. Q. (2010). Autopilots for small unmanned aerial vehicles: a survey. International Journal of Control, Automation, and Systems, 8(1), 36-44. https://doi.org/10.1007/s12555-010-0105-Z

Fahlstrom, P., \& Gleason, T. (2012). Introduction to UAV systems (4th ed.). New York: Wiley. https://doi.org/10.1002/9781118396780

Feng, G. (2006). A survey on analysis and design of model-based fuzzy control systems. IEEE Transactions on Fuzzy Systems, 14(5), 676-697. https://doi.org/10.1109/TFUZZ.2006.883415

Grytsenko, V., Volkov, O., Komar, M., \& Bogachuk, Y. (2018). Intelektualizatsiya suchasnykh system avtomatychnoho keruvannya bezpilotnymy lital' nymy aparatamy. Cybernetics and Computer Engineering Journal, 1(191), 45-59.

Johnson, E. N., \& Kannan, S. K. (2002, August). Adaptive flight control for an autonomous unmanned helicopter. Paper presented at the AIAA Guidance, Navigation, and Control Conference and Exhibit, Monterey, California (No. AIAA-2002-4439).

Kharchenko, V., Chepizhenko, V., Tunik, A., \& Pavlova, S. (2012). Avionika bezpilotnykh lital' nykh aparativ. Kiev: TOV Abrys-prynt.

Kumon, M., Udo, Y., Michihira, H., Nagata, M., Mizumoto, I., \& Iwai, Z. (2006). Autopilot system for Kiteplane. IEEE/ASME Transactions on Mechatronics, 11(5), 615-624. https://doi.org/10.1109/TMECH.2006.882994

Lopez, J., Dormido, R., Dormido, S., \& Gomez, J. P. (2015). A robust controller for an UAV flight control system. The Scientific World Journal, 2015. Articale ID 403236. https://doi.org/10.1155/2015/403236

Lopez, J., Dormido, R., Gomez, J. P., Dormido, S., \& Diaz, J. M. (2007, July 2-5). Comparison of H-infinity with QFT applied to an Altitude Command Tracker for an UAV. Paper presented at the Proceedings of the European Control Conference, Kos, Greece.

Moiseyev, V. S. (2013). Prikladnaya teoriya upravleniya bespilotnymi letatel' nymi apparatami: monografiya. Kazan: GBU Respublikanskiy tsentr monitoringa kachestva obrazovaniya.

Pavlov, V., \& Pavlova, S. (2015). Intellektual' noye upravleniye slozhnymi nelineynymi dinamicheskimi sistemami. Kiev: Naukova dumka.

Ross, T. J. (2004). Fuzzy logic with engineering applications (2nd ed.). New York: Wiley.

Shilov, K. (2014). Razrabotka sistemy avtomaticheskogo upravleniya bespilotnym letatel' nym apparatom mul'irotornogo tipa. Trudy MFTI, 4, 139-152. 